\title{
Defects in Lead Halide Perovskite Nanocrystals: Analogies and (Many) Differences with the Bulk
}

\author{
Stephanie ten Brinck, ${ }^{\dagger}$ Francesco Zaccaria, ${ }^{\dagger, \ddagger}$ and Ivan Infante ${ }^{* \dagger, \ddagger(0)}$
}

${ }^{\dagger}$ Department of Theoretical Chemistry, Vrije Universiteit Amsterdam, De Boelelaan 1083, 1081 HV Amsterdam, The Netherlands ${ }^{\ddagger}$ Department of Nanochemistry, Istituto Italiano di Tecnologia, Via Morego 30, 16163 Genova, Italy

Supporting Information

ABSTRACT: Understanding the origin of defects in lead halide perovskite nanocrystals is paramount to attaining long-term structural stability and improved optical efficiency, key features for their successful implementation in optoelectronic devices. Unlike other studies, we explore the possible formation of trap states in explicit, nonperiodic $\mathrm{CsPbBr}_{3}$ nanocrystal models about $3 \mathrm{~nm}$ in size. Using density functional theory, we compute the defect formation energies of interstitial, vacancy, and antisite defects in different regions of the nanocrystal (center, surface center, and surface edge), demonstrating that the most stable defect position is found at the surface. We ascribe the high defect

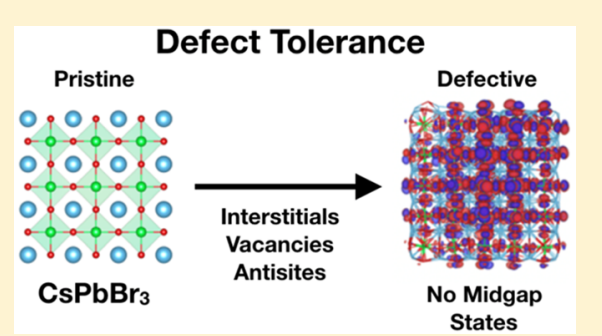
tolerance of $\mathrm{CsPBr}_{3}$ nanocrystals to the fact that vacancies, i.e. the loss of surface ligands as ion pairs, are energetically difficult to form and only excessive stripping of surface ligands might be problematic, as their detachment leaves undercoordinated $\mathrm{Br}^{-}$on the crystal surface that only in this case translates into deep traps.

$\mathrm{I}$ $\mathrm{n}$ recent years, researchers have grown increasingly interested in metal halide perovskites, in particular hybrid organic/inorganic and pure inorganic metal halides of the $\mathrm{APbX}_{3}$ form with $\mathrm{A}=\mathrm{CH}_{3} \mathrm{NH}_{3}{ }^{+}, \mathrm{Cs}^{+}$, etc. and $\mathrm{X}=\mathrm{Cl}, \mathrm{Br}$, or I. The nanoscale version of these materials presents unique features such as unity photoluminescence quantum yields (PLQYs), ${ }^{1,2}$ high defect tolerance, ${ }^{3,4}$ absorption in the visible spectrum $(400-800 \mathrm{~nm})$ that is tunable with the halide composition, $^{5-7}$ and narrow emission line widths (12-42 $\mathrm{nm}) .{ }^{6}$ For these reasons, they have become potential candidates for a wide variety of optoelectronic applications such as lasing, ${ }^{8-10}$ light-emitting diodes (LEDs), ${ }^{11-16}$ and photovoltaics. $^{17,18}$

One of the main reasons perovskites have outstanding optoelectronic characteristics is their high defect tolerance, especially in thin films of bulk methylammonium lead iodide that have demonstrated outstanding performance for photovoltaics with power conversion efficiencies up to $25 \% .{ }^{19}$ Defect tolerance in bulk perovskites has often been studied computationally, ${ }^{2,4,20,21}$ however, as it will be shown in this Letter, not as extensively for (fully inorganic) perovskite nanocrystals (NCs). One of the main differences between bulk and NCs emerges immediately when looking at the typical defect densities of bulk perovskites, measured (and computed) in the order of $10^{11}-10^{16} \mathrm{~cm}^{-3} \cdot{ }^{20,22,23}$ In the nanoscale regime, this corresponds to about one defect in approximately $10^{2}-10^{7}$ for a perovskite NC $10 \mathrm{~nm}$ in size. These values are too low to explain the frequent loss of photoluminescence efficiency that affects NC colloids (more than 50\% after purification procedures). One explanation for this is that the energetics of defect formation in NCs is expected to change dramatically compared to the bulk, mostly for two reasons: (1) the presence of a complex chemical equilibrium at the NC-ligands-solvent interface that can expose defects to a different environment than in the bulk and (2) the location of the defect, which is more likely to be found at the NC surface instead of the core because of the large number of surface atoms. Regarding the former point, chemical equilibria at the NC surface alter structural stability of the NC, which is still a major drawback for NC colloids and is mostly linked to desorption of surface ligands that can occur during washing or diluting. ${ }^{24}$ Ligand detachment can lead to NC aggregation and precipitation and, more importantly, to exposure of defects at the surface. While methods to restore the PLQY are continuously developed, mostly by post-treating the NC surface with a new set of ligands, the exact mechanism by which defects form and PL efficiency is lost is not yet fully understood. In addition, the nanometric size of the crystallite (about $8-10 \mathrm{~nm}$ ) suggests that, even if bulk-type defects are formed in the core, they could eventually travel toward the surface if they are more stable in this region. This suggests that carriers might always be trapped at the surface. This is not surprising because major losses are known to occur at the grain boundaries of perovskite

Received: September 5, 2019

Accepted: October 15, 2019

Published: October 15, 2019 
thin films in solar cell devices, and strategies to efficiently passivate this region are widely studied to improve power conversion efficiencies. ${ }^{25}$

Density functional theory (DFT) is a powerful tool to provide meaningful insight into the surface chemistry of NCs. It is crucial to perform computations on realistic NC models to capture how defects form and to assess the driving force (energetics) of their formation. Providing a better understanding of the chemistry of defects and comparing them to the bulk will help develop new strategies to prevent crystal degradation and suppress the formation of trap states, as well as to maintain high PLQY over longer periods of time. Theoretical work that involves realistic NC models has been, however, notoriously scarce, mostly because the NC surface is a complex region to model, as both ligands and facet composition need to be explicitly accounted for and assessed against experiments. In this study, we employ DFT to study the formation of defects in NCs. First, we draw a comparison between bulk and NC defects; we then evaluate the effects of various types of defects on the electronic structure of explicit, nonperiodic NC models. We demonstrate that most of the defects require large formation energies (vacancies and antisites) with the exception of interstitial defects, which are energetically favorable. Even so, the most stable interstitials deliver shallow traps, affecting the electronic structure of the material only slightly. We can ascribe the large defect tolerance of perovskite NCs to the resilience to form deep traps even after vacancies are formed at the surface. Such vacancies need to be formed in large amounts before they can actually transform into trap states, a requirement that is met in ordinary experimental conditions only after purification procedures or with the employment of ligands that can desorb easily from the surface.

In this work, defects are classified and named according to IUPAC notation as ${ }^{26} A_{B}^{C}$, where $A$ is the species ( $V$ for a vacancy or the atom name for an added species to the lattice), $\mathrm{B}$ the lattice position that the A species occupies, and $C$ the charge of the lattice site occupied by species A. We will focus on fully inorganic $\mathrm{Cs} \mathrm{PbBr}_{3} \mathrm{NCs}$, as these are considered the benchmark NCs for a variety of reasons: for example, highest optical purity among the halide group, stable crystalline phase, and tolerance against various surface treatments. To draw a meaningful comparison between the formation of defects in periodic $\mathrm{CsPbBr}_{3}$ and our NC models, we briefly explain how defect formation energies are estimated in the two cases. In bulk materials, the defect formation energy (DFE) is calculated according to the following formula: ${ }^{20,27,28}$

$$
\begin{aligned}
& \operatorname{DFE}(\alpha, q)=E(\alpha, q)-E(\text { bulk }) \\
& \quad-\sum_{\mathrm{A}} n_{\mathrm{A}} \mu_{\mathrm{A}}+q\left(E_{\mathrm{F}}+E_{\mathrm{VBM}}+\Delta V\right)+E^{q}
\end{aligned}
$$

where $E(\alpha, q)$ is the total energy of the supercell containing the defect $\alpha$ with charge $q, E$ (bulk) the total energy of the supercell containing the pristine system without the defect, $n_{\mathrm{A}}$ the number of atoms involved in creating the defect, $\mu_{\mathrm{A}}$ the atomic chemical potential in reference to the total energy of the pure elements composing the lattice, $E_{\mathrm{F}}$ the Fermi energy of the nondefective system, $E_{\mathrm{VMB}}$ the valence band maximum, $\Delta V$ the electrostatic shift induced by the defect in the charged supercell, and $E^{q}$ the background electrostatic charge of the supercell that prevents the charge of the defect from being periodically replicated.
As explained in more detail by Meggiolaro et al., ${ }^{20}$ the quality of the bulk calculations depends on several factors, like the accuracy of (1) the electrostatic background correction, (2) the electrostatic shift induced by the defect, (3) the chemical potential of the elemental constituting species, and (4) the type of DFT exchange-correlation approximation employed (semilocal, hybrid or GW, with or without spinorbit coupling). In the framework of bulk defect formation, the chemical potential of the defective species, such as solid $\mathrm{Pb}$, solid Cs, or molecular bromine $\mathrm{Br}_{2}$, needs to be explicitly calculated. While this approach of computing the energy of defect formation is effective for bulk materials, it is not without problems when applied to NCs. First, perovskite NCs are typically prepared and dispersed in solution, meaning that when a defect is formed, its chemical potential is not related to the formation of solid precipitates. Rather, we expect that the creation of defects is driven by the formation of molecular ion pairs in solution, such as $\mathrm{CsBr}$ and $\mathrm{PbBr}_{2}$. These ion pairs are responsible for the creation of vacancy (when removed) or interstitial (when added) defects in NCs. For example, if $\mathrm{a} \mathrm{V}_{\mathrm{Pb}}^{2-}$ vacancy is created, then its formation energy should be referenced to the detachment of a neutral $\mathrm{PbBr}_{2}$ species, which is then dispersed in the solvent. This is the most likely mechanism to take place in the chemical environment in which the NC was prepared, which is usually a low dielectric solvent, e.g., toluene. Note that the creation of the lead defect is accompanied by the removal of $\mathrm{Br}$ ions from the lattice; thus, the defect is more correctly described as $V_{\mathrm{Pb}}^{2-}+2 \mathrm{~V}_{\mathrm{Br}}^{+}$.

Summarizing, to calculate the DFE in our NC models, we will thus use the following simplified equation for the defects:

$$
\operatorname{DFE}(\alpha)=E(\mathrm{NC}(\alpha))-E(\mathrm{NC}) \pm E(\mathrm{~s})
$$

where $E(\mathrm{NC}), E(\mathrm{NC}(\alpha))$, and $E(\mathrm{~s})$ are the total energies of the pristine $\mathrm{NC}$, the NC with the defect $\alpha$ which is characterized by the neutral form s, and the energy of the neutral species s, respectively. For example, in the case of the $\mathrm{V}_{\mathrm{Pb}}^{2-}$ vacancy of the previous example, the defect formation energy is expressed as

$$
\begin{aligned}
& \operatorname{DFE}\left(\mathrm{V}_{\mathrm{Pb}}^{2-}+2 \mathrm{~V}_{\mathrm{Br}}^{+}\right) \\
& \quad=E\left(\mathrm{NC}\left(\mathrm{V}_{\mathrm{Pb}}^{2-}+2 \mathrm{~V}_{\mathrm{Br}}^{+}\right)\right)-E(\mathrm{NC}) \pm E\left(\mathrm{PbBr}_{2}\right)
\end{aligned}
$$

By treating the defect formation process in this manner, the above equations are fully balanced, charge neutrality is maintained at any point of the calculation, and no electrostatic shift is needed to correct for the energy levels. In addition, there is also no need for background charge correction because the whole NC is explicitly included in the calculation. Finally, while not explicitly done in this work, the $E_{\mathrm{VBM}}$ can easily be estimated by computing the ionization potential of the neutral system in a nonperiodic calculation. This has the advantage of pinning the exact position of the valence band maximum (VBM) and conduction band minimum (CBM), with the latter from an excitation energy (or a simple HOMO-LUMO gap) calculation. In this way, it could also be possible to assess the propensity of the system to be $n$ - or p-doped. We anticipate that in a low-dielectric environment, which is usually the one used in the synthesis, the NC model systems considered here are usually intrinsic.

$\mathrm{NC}$ models of $\mathrm{CsPbBr}_{3}$ of $2.9 \mathrm{~nm}$ in the lateral side were constructed by cutting the cubic bulk (space group $P m \overline{3} m$ ) along the (100) directions. Assuming all atoms maintain their thermodynamically most stable oxidation state, i.e., Cs as +1 , 


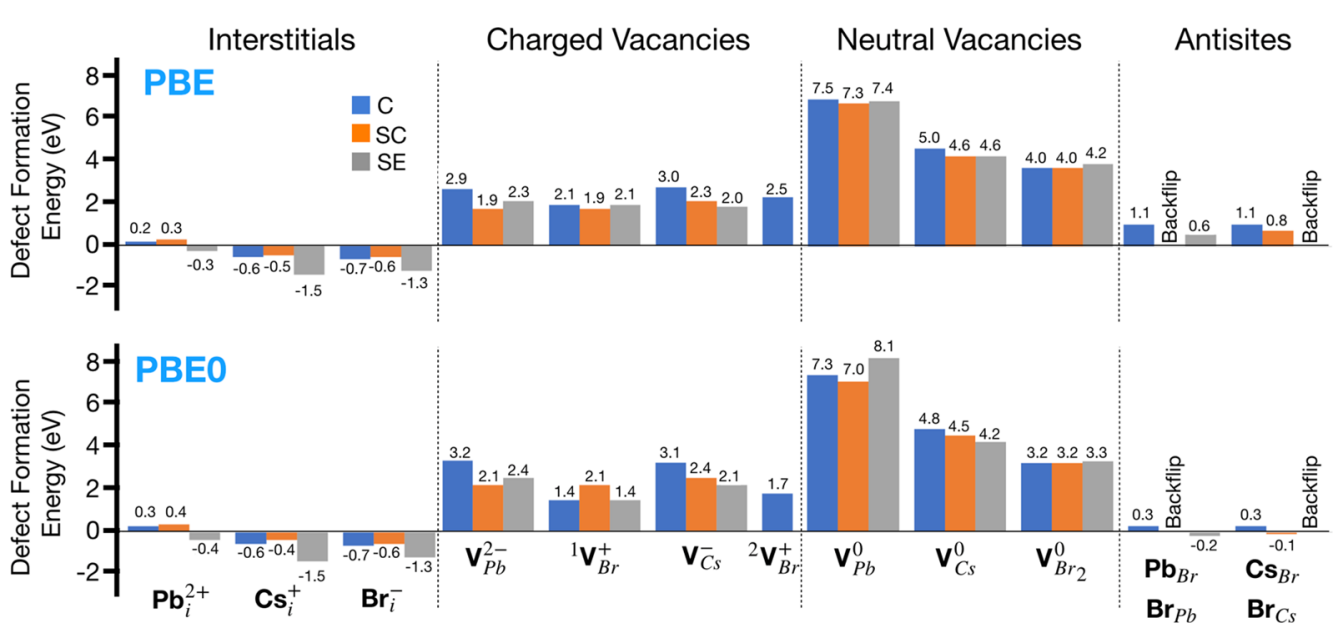

Figure 1. Defect formation energies (DFEs) associated with interstitial, charged, neutral vacancies, and antisite substitutions listed in electronvolts. The DFEs are computed following geometry optimizations carried out at the DFT/PBE0 level of theory. Backflip means that the antisite displacement goes back to its initial nondefective configuration. Note that here we report only the defect name without the counterion. The full name of the defect is presented in Table 1.

Table 1. Type of Defect, Counterion, and Full Defect Name for All Defects Studied

\begin{tabular}{|c|c|c|c|c|c|c|c|c|c|c|c|c|}
\hline \multirow[b]{2}{*}{ defect } & \multicolumn{3}{|c|}{ interstitials } & \multicolumn{4}{|c|}{ charged vacancy } & \multicolumn{3}{|c|}{ neutral vacancy } & \multicolumn{2}{|c|}{ antisite } \\
\hline & $\mathrm{Pb}_{\mathrm{i}}^{2+}$ & $\mathrm{Cs}_{\mathrm{i}}^{+}$ & $\mathrm{Br}_{\mathrm{i}}^{-}$ & $\mathrm{V}_{\mathrm{Pb}}^{2-}$ & ${ }^{1} \mathrm{~V}_{\mathrm{Br}}^{+}$ & $\mathrm{V}_{\mathrm{Cs}}^{-}$ & ${ }^{2} \mathrm{~V}_{\mathrm{Br}}^{+}$ & $\mathrm{V}_{\mathrm{Pb}}^{0}$ & $\mathrm{~V}_{\mathrm{Cs}}^{0}$ & $\mathrm{~V}_{\mathrm{Br} 2}^{0}$ & $\mathrm{~Pb}_{\mathrm{Br}}=\mathrm{Br}_{\mathrm{Pb}}$ & $\mathrm{Cs}_{\mathrm{Br}}=\mathrm{Br}_{\mathrm{Cs}}$ \\
\hline counterion & $\mathrm{Br}_{\mathrm{Br}}^{-}$ & $\mathrm{Br}_{\mathrm{Br}}^{-}$ & $\mathrm{Cs}_{\mathrm{Cs}}^{+}$ & $\mathrm{V}_{\mathrm{Br}}^{+}$ & $\mathrm{V}_{\mathrm{Pb}}^{2-}$ & $\mathrm{V}_{\mathrm{Br}}^{+}$ & $\mathrm{V}_{\mathrm{Cs}}^{-}$ & & & & & \\
\hline full defect name & $\mathrm{Pb}_{\mathrm{i}}^{2+}+2 \mathrm{Br}_{\mathrm{Br}}^{-}$ & $\mathrm{Cs}_{\mathrm{i}}^{+}+\mathrm{Br}_{\mathrm{Br}}^{-}$ & $\mathrm{Br}_{\mathrm{i}}^{-}+\mathrm{Cs}_{\mathrm{Cs}}^{+}$ & $\mathrm{V}_{\mathrm{Pb}}^{2-}+2 \mathrm{~V}_{\mathrm{Br}}^{+}$ & $\mathrm{V}_{\mathrm{Pb}}^{2-}+2^{1} \mathrm{~V}_{\mathrm{Br}}^{+}$ & $\mathrm{V}_{\mathrm{CS}}^{-}{ }^{2}{ }^{2} \mathrm{~V}_{\mathrm{Br}}^{+}$ & $\mathrm{V}_{\mathrm{Cs}}^{-} t^{2} \mathrm{~V}_{\mathrm{Br}}^{+}$ & $\mathrm{V}_{\mathrm{Pb}}^{0}$ & $\mathrm{~V}_{\mathrm{Cs}}^{0}$ & $\mathrm{~V}_{\mathrm{Br} 2}^{0}$ & $\mathrm{~Pb}_{\mathrm{Br}}=\mathrm{Br}_{\mathrm{Pb}}$ & $\mathrm{Cs}_{\mathrm{Br}}=\mathrm{Br}_{\mathrm{Cs}}$ \\
\hline
\end{tabular}

$\mathrm{Pb}$ as $2+$, and $\mathrm{Br}$ as -1 , charge neutrality of the model was achieved by removing the excess positive charge, as this approach proved to be the most resilient to the formation of localized states at the surface. ${ }^{4}$ Any residual charge was compensated by removing $\mathrm{Cs}^{+}$from the edges and vertices of the NC, as these are the least strongly bound positions in the NC. ${ }^{4}$ The molar ratio of $\mathrm{Cs}: \mathrm{Pb}: \mathrm{Br}$ of this pristine NC model was determined to be roughly $1.6: 1: 3.6$, which is in agreement with experimentally observed Br-rich nanocrystals. ${ }^{29,30}$ In our model, the $\mathrm{Cs}: \mathrm{Pb}$ ratio is overestimated against the experiments because at least half of the Cs at the surface, in the standard synthesis, is known to be replaced by alkylammonium ligands. For computational efficiency and for avoiding further complications in our study, only Cs ions were included in our model. These organic ligands have a wide band gap and do not significantly alter the electronic structure of $\mathrm{CsPbBr}_{3} \mathrm{NCs}^{4}{ }^{4} \mathrm{To}$ explore the effect of defects, we first relaxed a pristine, defectfree NC, see ref 4 , until an energetic minimum configuration is reached. This optimized NC model is then used as a starting structure to introduce the defective models. Using the CP2k program, ${ }^{31}$ the computed band gap was 2.4 and $3.7 \mathrm{eV}$ for $\mathrm{DFT} / \mathrm{PBE}^{32}$ and $\mathrm{DFT} / \mathrm{PBE} 0{ }^{33}$ respectively. While PBE is in better agreement with experiments, this result is assigned to an accidental cancelation of errors due to the lack of the spinorbit coupling term. More details on the computational method are given in the Supporting Information.

Unlike the bulk, each defective system presents different energetics depending on the position of the defect, either in the core or at the surface (center or edge), and on the location of the counterions that neutralize the charge. The counterions are always kept in the same position at the surface: for vacancies it will correspond to the elimination of one or two surface ions (depending on the defect), while for interstitial defects, where species are added, the counterions are added at the surface in its natural position of the lattice. In IUPAC notation, this latter addition is defined as, e.g. $\mathrm{Br}_{\mathrm{Br}}^{-}$for an added $\mathrm{Br}^{-}$counterion. Overall, we explored three different defect/ counterion combinations for each type of defect: core/surface (C), surface-center/surface (SC), and surface-edge/surface (SE). The DFEs for all type of defects are shown in Figure 1 and schematically depicted in each figure that follows. The full defect names are listed in Table 1. The relaxed structures of the defective systems are illustrated in Figures S1-S4. Note that the charge of a "charged vacancy" refers to the charge of the point defect without the inclusion of the counterion. This is done to maintain a fair comparison with the nomenclature used in the bulk.

For all interstitial defective systems, the cationic species $\left(\mathrm{Cs}_{i}^{+}\right.$and $\left.\mathrm{Pb}_{\mathrm{i}}^{2+}\right)$ were placed in the same plane as $\mathrm{Cs}^{+}$and $\mathrm{Br}^{-}$ (see schematics in Figure 2A), either at the core or at the surface.

Inspection of Figure 1 shows that for all cases, interstitial positions are favored energetically. In Figure $2 \mathrm{~B}$, we present the electronic structure for all interstitials explored. Interstitial positions generated upon the addition of $\mathrm{CsBr}$ or $\mathrm{PbBr}_{2}$ species are always favored energetically, implying that the NC can easily sustain the addition of either $\mathrm{CsBr}$ or $\mathrm{PbBr}_{2}$ ion pairs in the lattice. In the experiments, this means that the NC tends to accommodate metal salts if these are available from the environment. Postsynthetic treatment of the NC by adding ion pairs can thus be very effective for these materials, as also demonstrated in many experimental works. ${ }^{34-36}$

Addition of neutral species can lead to the occupation of interstitial positions from either the cation $\left(\mathrm{Cs}_{\mathrm{i}}^{+}\right.$or $\left.\mathrm{Pb}_{\mathrm{i}}^{2+}\right)$ or the anion $\left(\mathrm{Br}_{\mathrm{i}}^{-}\right)$. The most favorable additions are usually at the surface edge, which is the most reactive region of the NC. Interstitials at the core are unlikely to form as they are less favorable energetically. When possible, though, the NC tries to reorganize itself to accommodate each ion in its natural position in the crystal lattice, eliminating the defect. A case in 
A)

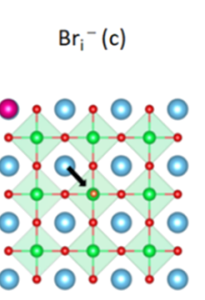

Top View

$\mathrm{Br}_{\mathrm{i}}^{-}$(se)

$\mathrm{Br}_{\mathrm{i}}{ }^{-}$(sc)

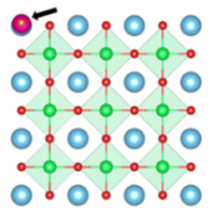

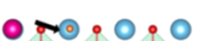

$-0-0-0-0$

0.0 .0 .0

- 0.0000

0.0 .0 .0

0.0 .0$.

0.0 .0 .0

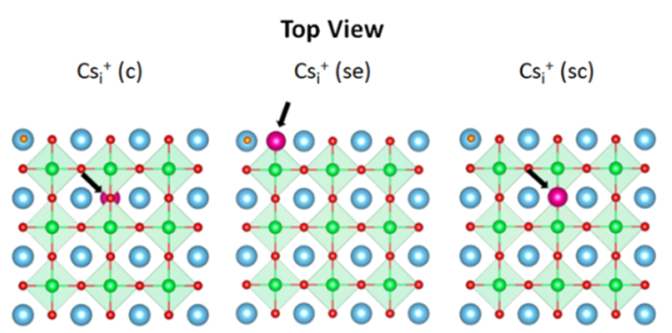

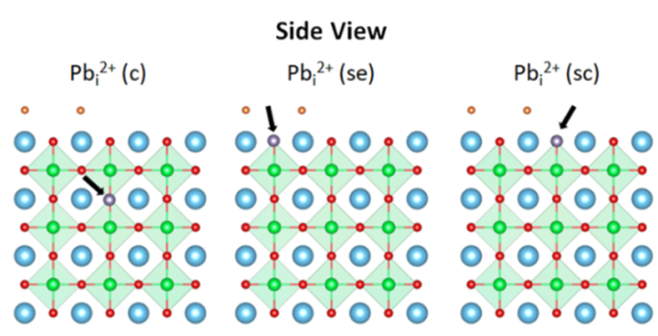

B)

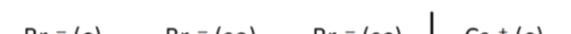

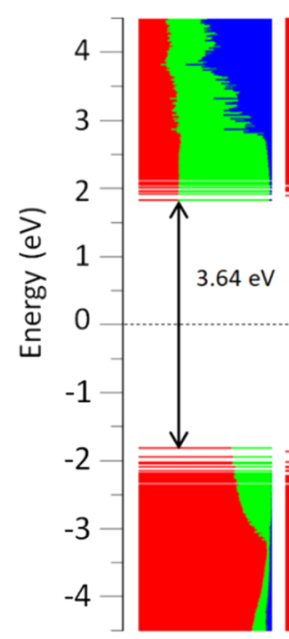

$\operatorname{DFE}(\mathrm{eV})=-0.7$
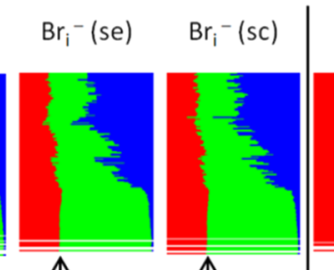

$\mathrm{Cs}_{\mathrm{i}}{ }^{+}(\mathrm{c})$
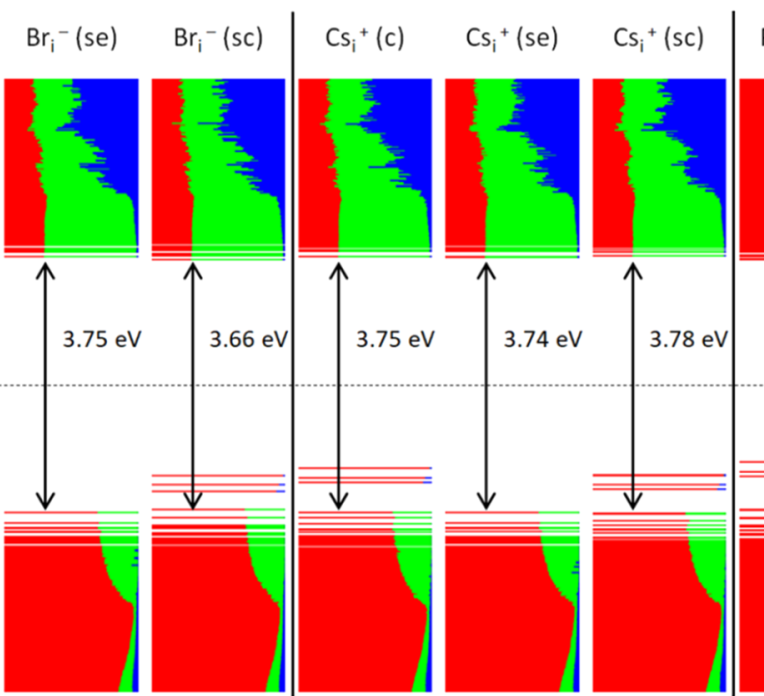

$-1.3$
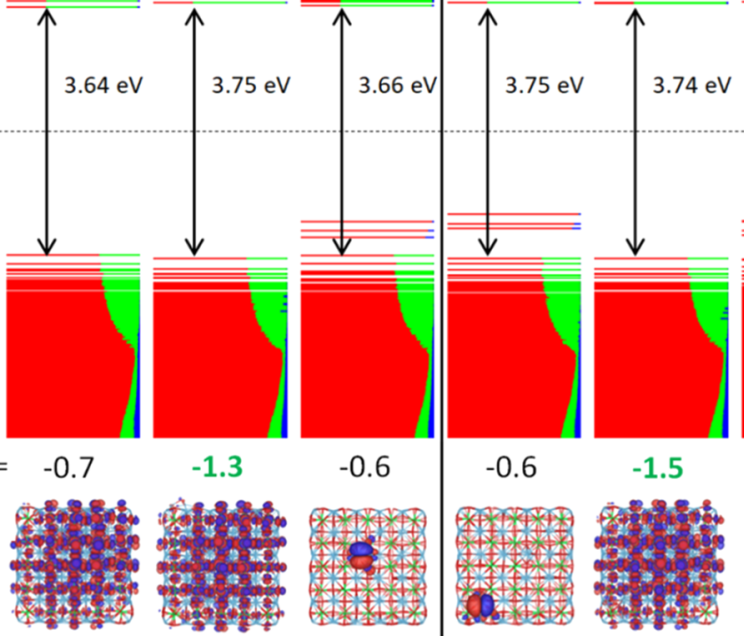

$-0.6$
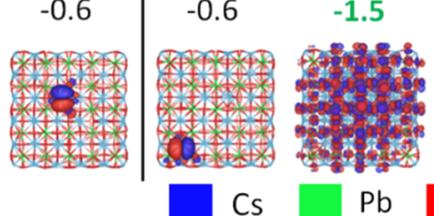

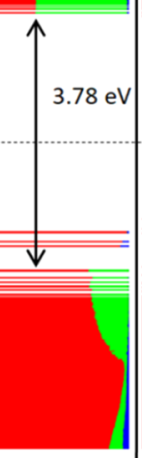

$-0.4$

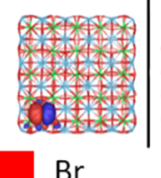

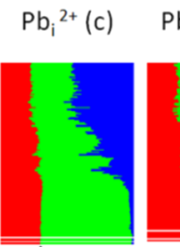
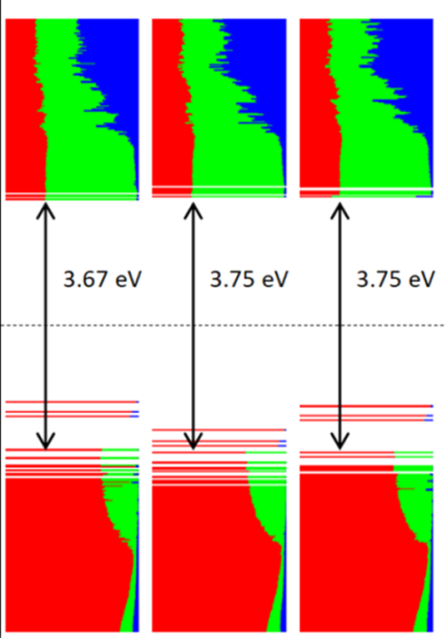

0.3
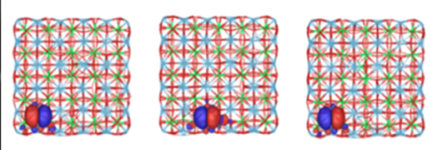

Figure 2. (A) Schematic depiction of the interstitial defects in different areas of the NC shown in top and side views. (B) Density of states (DOS) plots belonging to each of these systems calculated at DFT/PBE0 level of theory, with the dotted line indicating the Fermi energy $\left(E_{\mathrm{F}}\right)$. Each line in the plot represents a molecular orbital (MO) and the color of the line represents the contribution of $\mathrm{Cs}(\mathrm{blue}), \mathrm{Pb}(\mathrm{green})$, and $\mathrm{Br}$ (red). Shown below this plot are the defect formation energies (DFEs) and the highest occupied molecular orbital (HOMO) belonging to each system.

point is the most favorable DFE of $\mathrm{Cs}_{\mathrm{i}}^{+}(-1.3 \mathrm{eV})$ where the added Cs ion in interstitial position moves on the NC surface to occupy a Cs site left vacant during the process of chargebalancing the NC. Because it is a natural position for Cs, i.e. it transforms from $\mathrm{Cs}_{i}^{+}$to $\mathrm{Cs}_{\mathrm{Cs}}^{+}$, we do not consider this as an interstitial defect. Indeed, also the electronic structure is trapfree (see DOS for $\mathrm{Cs}_{\mathrm{i}}^{+}(\mathrm{se})$ ). A similar mechanism occurs for the most stable $\mathrm{Br}_{\mathrm{i}}^{-}$(DOS for $\mathrm{Br}_{\mathrm{i}}^{-}(\mathrm{se})$ ), which reconstructs on the surface to attain the natural position of $\mathrm{Br}_{\mathrm{Br}}^{-}$. On the other hand, the most stable interstitial positions of $\mathrm{Pb}_{\mathrm{i}}^{2+}$ remains interstitial and presents localized states right above the valence band maximum (see DOS for $\mathrm{Pb}_{\mathrm{i}}^{+}(\mathrm{se})$ ). Interestingly, these localized states belong to the $4 \mathrm{p}$ orbitals of the $\mathrm{Br}$ counterions used to compensate the charge of the $\mathrm{Pb}_{\mathrm{i}}^{2+}$ defect and not to the $\mathrm{Pb}$ ion itself. We noticed that whenever midgap states are formed, they can emerge only from the counterion (halide), regardless of the type of interstitial defect (cationic or anionic). Moreover, the trap state is formed only when such a halide ion occupies surface positions that point outward the surface. In other words, if the added bromide is in excess against the available halide surface sites, then its lone pairs will be dangling and nonbonding, forming trap states. This situation can occur, for example, when the perovskite NC is already terminated by the CsBr layer and excess CsBr (or any other Cs salt) is added to the NC.

Vacancy defects are created by removing an ion $\left(\mathrm{Cs}^{+}, \mathrm{Pb}^{2+}\right.$, $\mathrm{Br}^{-}$) from either the core or the surface of the model (Figure $3 \mathrm{~A})$. The overall charge neutrality of the system is again 
A)

Side View
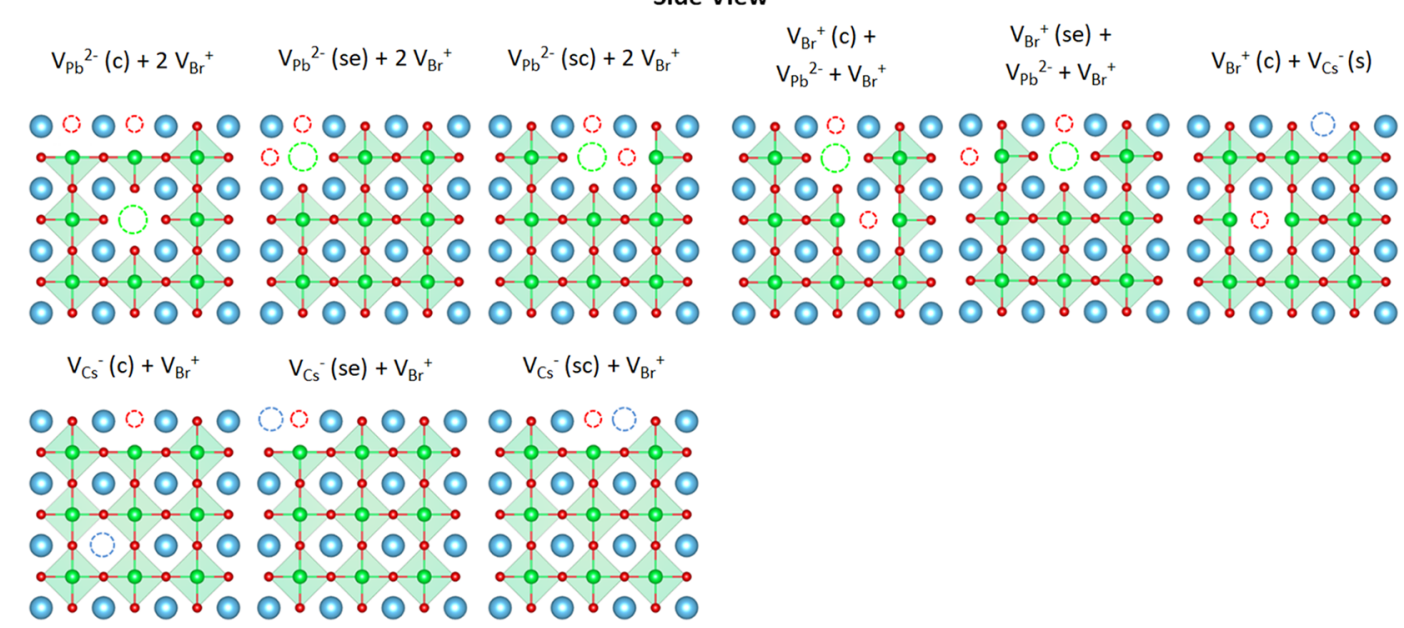

B)

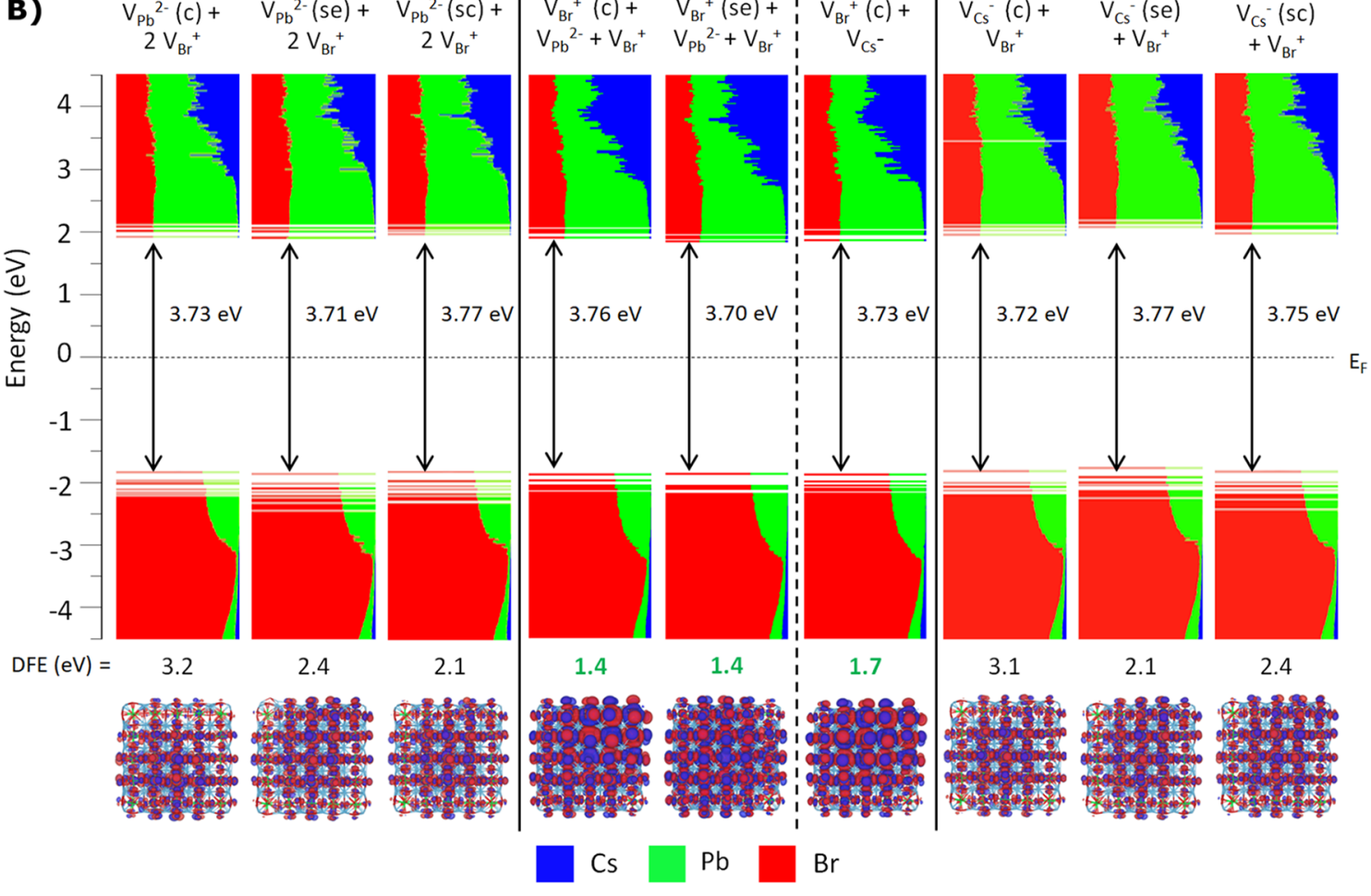

Figure 3. (A) Schematic depiction of the vacancy defects in different areas of the NC shown in the side view. (B) DOS plots belonging to each of these systems calculated at the DFT/PBE0 level of theory, with the dotted line indicating the Fermi energy $\left(E_{\mathrm{F}}\right)$. Each line in the plot represents a molecular orbital (MO) and the color of the line represents the contribution of $\mathrm{Cs}$ (blue), $\mathrm{Pb}$ (green), and $\mathrm{Br}$ (red). Shown below this plot are the DFEs and the HOMO belonging to each system.

maintained by removing additional counterions from the surface and also when the vacancy is created at the core. Inspection of Figure 1 reveals that the formation of vacancies is always endothermic. The energy required to create vacancies is in the range of $1.4-2.3 \mathrm{eV}$ depending on the position of the vacancy in the lattice. This corresponds to negligible vacancy (defect) densities. It is thus expected that removal of metal salts from the NC must be induced from other species present in the environment. This is indeed what happens when, for example, an excess of amines is added to a $\mathrm{CsPbBr}_{3} \mathrm{NC}$ solution. Primary alkylamine molecules induce the extraction of $\mathrm{PbBr}_{2}$ from the $\mathrm{NC}$, which then transforms into $\mathrm{OD}$ $\mathrm{Cs}_{4} \mathrm{PbBr}_{6} \mathrm{NCs}^{37-40}$ In less harsh environments, the detachment of these metal halide salts may be induced during washing by the solvent itself.
The extraction of metal salts, i.e. the formation of a neutral vacancy, is more effective at the surface, whereas creating the vacancy in the $\mathrm{NC}$ core is the least energetically favorable. Regardless of their position and type, vacancies have little effect on the electronic structure of the NC and do not form any traps, as highlighted in Figure $3 \mathrm{~B}$. In a previous paper, some of us highlighted this aspect by proving that midgap states stem from undercoordinated halide ions that are created only when a large number of surface $\mathrm{CsBr}$ moieties are removed from the outer layer of the perovskite NC lattice. ${ }^{2}$ This situation is difficult to attain because of the high energy required to create these vacancies and can explain the high defect tolerance of perovskite NCs. On the other hand, NCs prepared in the experiments are less ideal: the surface is terminated by metalorganic salts, e.g., Cs(carboxylate), 
A)
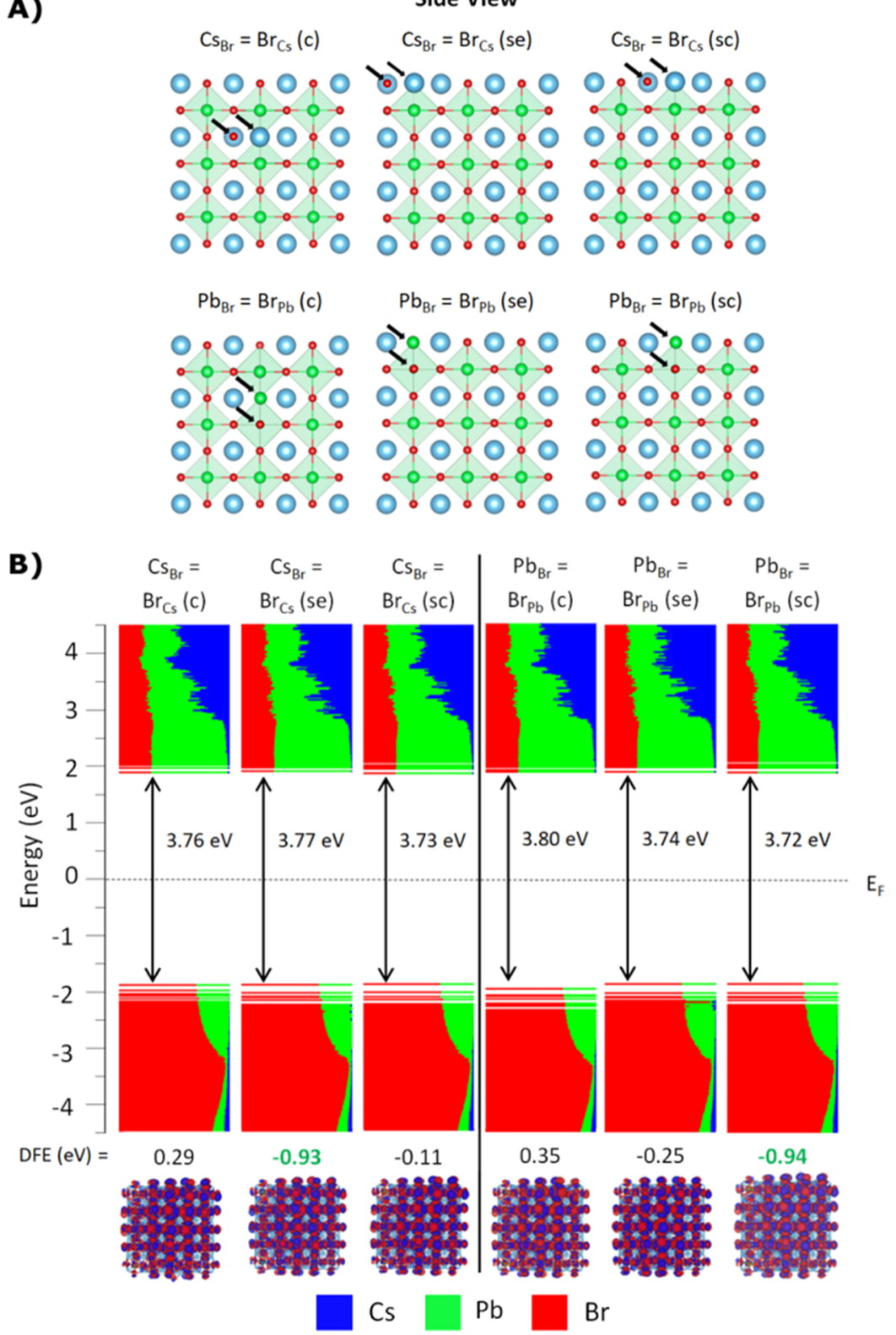

Figure 4. (A) Schematic depiction of the antisite defects in different areas of the NC shown in the side view. (B) DOS plots belonging to each of these systems calculated at the DFT/PBE0 level of theory, with the dotted line indicating the Fermi energy $\left(E_{\mathrm{F}}\right)$. Each line in the plot represents a MO, and the color of the line represents the contribution of $\mathrm{Cs}$ (blue), $\mathrm{Pb}$ (green), or $\mathrm{Br}$ (red). Shown below this plot are the DFEs and the HOMO belonging to each system.

alkylammonium bromide, rather than the simple $\mathrm{CsBr}$ termination used in the calculations. These capping species usually have a moderate solubility in the organic solvent used in the synthesis or during the NC purification, e.g., octadecene and toluene, thus altering the NC-ligand binding affinity. In such an environment, the loss of ligands, and thus the formation of vacancies, is more likely, especially after purification. Another mechanism that helps in the detachment of metalorganic salts from the NC surface is the neutralization mechanism. ${ }^{1}$ Here, the detached oleylammonium bromide can transform into oleylamine and $\mathrm{HBr}$. $\mathrm{HBr}$ is volatile and can escape the environment, shifting the reaction toward the elimination of metal salts or ion pairs from the NC surface. This can generate a large number of surface vacancies, which can then expose highly undercoordinated halide ions, thus introducing trap states and reducing the PL efficiency.
In some cases, the lattice can expel some of the ions in their elemental form, i.e. as $\mathrm{Cs}(0), \mathrm{Pb}(0)$ or molecular halogen $\mathrm{Br}_{2}$, implying a redox reaction occurring in the NC (Figure 4A). The elimination of neutral $\mathrm{Pb}(0)$ and $\mathrm{Cs}(0)$ means that these ions are reduced; hence, the NC is oxidized (p-doping). In contrast, the elimination of $\mathrm{Br}_{2}$ implies the reduction of the NC (n-doping). We explored the energetics of these mechanisms and realized that they are all strongly endothermic, regardless of the position of the extracted ion (see Figure 4B). Defect densities are very low, indicating a very low probability of formation for this type of defect. In some cases, this process can be triggered remotely in harsh conditions. A typical example is the formation of clusters of metal $\mathrm{Pb}(0)$ on top of the $\mathrm{CsPBr}_{3}$ by exposing a film of perovskite NCs under a TEM beam (for example, Figure 1 in ref 36). Other examples can include oxidation by air, when the 
A)

\begin{tabular}{|c|c|c|c|c|c|}
\hline \multicolumn{6}{|c|}{ Side View } \\
\hline $\mathrm{Cs}^{0}(\mathrm{c})$ & $\mathrm{Cs}^{0}(\mathrm{se})$ & $\mathrm{Cs}^{0}(\mathrm{sc})$ & $\mathrm{Pb}^{0}(\mathrm{c})$ & $\mathrm{Pb}^{0}$ (se) & $\mathrm{Pb}^{0}(\mathrm{sc})$ \\
\hline $\begin{array}{cccccc}0 & 0 & 0 & 0 & 0 \\
0 & 0 & 0 & 0 & 0 & 0 \\
0 & 0 & 0 & 0 & 0 & 0 \\
0 & 0 & 0 & 0 & 0 & 0 \\
0 & 0 & 0 & 0 & 0 & 0 \\
0 & 0 & 0 & 0 & 0 & 0 \\
0 & 0 & 0 & 0 & 0 & 0\end{array}$ & 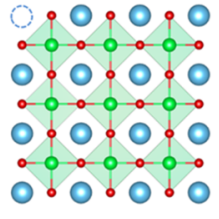 & 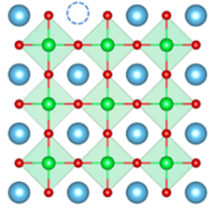 & 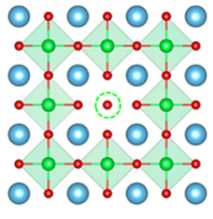 & 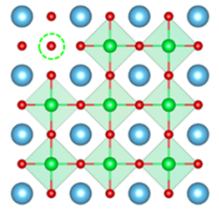 & 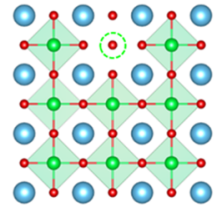 \\
\hline $\mathrm{Br}^{0}(\mathrm{c})$ & $\mathrm{Br}^{0}(\mathrm{se})$ & $\mathrm{Br}^{0}(\mathrm{sc})$ & . 2 (1) & 0.70 & - 210 \\
\hline 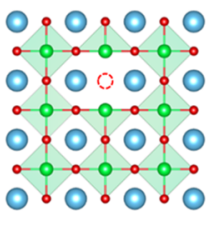 & 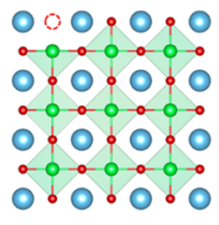 & 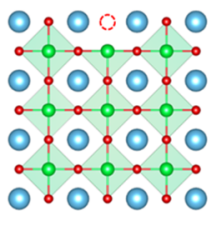 & 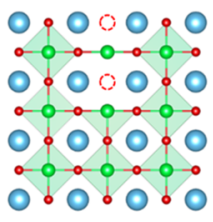 & 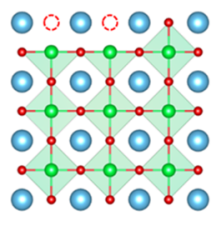 & 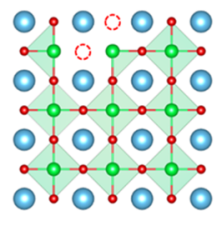 \\
\hline
\end{tabular}

B)

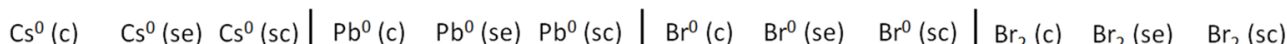

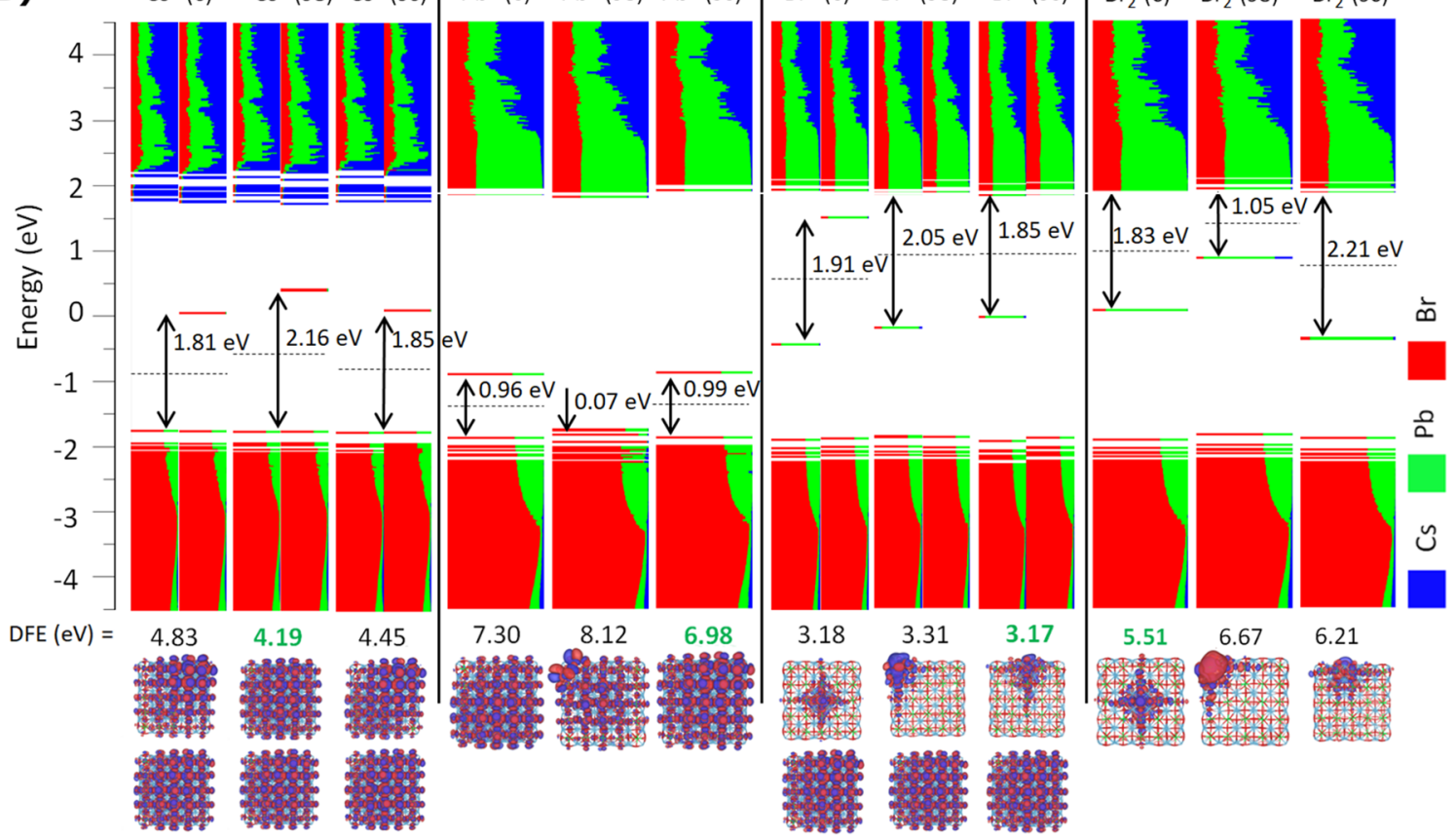

Figure 5. (A) Schematic depiction of the neutral defects in different areas of the NC shown in the side view. (B) DOS plots belonging to each of these systems calculated at the DFT/PBE0 level of theory, with the dotted line indicating the Fermi energy $\left(E_{\mathrm{F}}\right)$. Each line in the plot represents a molecular orbital (MO), and the color of the line represents the contribution of Cs (blue), $\mathrm{Pb}$ (green), or $\mathrm{Br}$ (red). Shown below this plot are the DFEs and the HOMO belonging to each system. Note that some structures are open-shell and have been relaxed to their most stable spin configuration.

valence band energy levels move toward the vacuum, and also via remote or electrochemical doping. In typical perovskite NC synthesis, these conditions are usually not met.

Another type of defect typically studied in the bulk is the antisite, i.e. where a cation exchanges position with an anion. For the NCs presented in this work, we calculated the swap between $\mathrm{Pb}$ with $\mathrm{Br}$ and $\mathrm{Cs}$ with $\mathrm{Br}$ (see Figure $5 \mathrm{~A}$ ). We noticed that, unlike the core, the antisites behave unexpectedly at the surface. Starting from a surface antisite position and upon geometry optimization, the structure reorganizes to a nonantisite configuration; in some cases, the ions backflip to their initial position. For example, $\mathrm{Pb}$ just moves back at the center of the octahedron; in other cases, the ions reorganize, forming stable $\mathrm{PbBr}_{3}{ }^{-}$units at the surface, which are energetically favorable (Figure S5). We are not completely sure if the formation of these lead bromide units is an artifact from the calculations considering that the surface is more flexible and allows for large geometrical reorganizations, hindered at the core. Regardless of this, as expressed in Figure $5 \mathrm{~B}$, the formation of these subunits still forms shallow traps, without significantly altering the electronic structure of the nanocrystal.

As explained in the previous sections, the nature of defects in bulk perovskites and NCs can be rather different. While a direct comparison with the bulk defect formation energies is difficult to draw (different DFT methodology, presence or absence of spin-orbit coupling, and localized basis-set versus plane-waves), we can observe that in both cases shallow traps have low formation energies, whereas deep traps require more energy. In the bulk, even with low defect densities, charged and neutral defects can form and be stabilized by the surroundings. Such defects can travel inside the bulk for extended periods of 
time affecting the recombination and the extraction of carriers, and as such the film conductivity, the photoluminescence efficiency and other important macroscopic characteristics. At the nanoscale, on the other hand, we expect that defects that form midgap states are mostly located at the surface for energetic reasons and because the distance that a defect needs to travel from the core to the surface is much shorter compared to the bulk. This implies that even if the defect is formed upon photoexcitation in the core of the NC, the carriers can still be trapped at the surface within the emission lifetime. A point in common between the bulk and the NCs is that the NC surface can resemble the grain boundaries of perovskite films, which can present characteristics similar to the ones discussed for NCs. For this reason, successful strategies used to passivate the NCs surface can be transferred to the bulk to fix defects at the grain boundaries or vice versa.

It is customary in the NC field to describe the energetics of defects in the same way as for the bulk. However, this approach can be largely misleading in many cases, as demonstrated in this work. For example, the typical understanding that halide-deficient NCs introduce midgap states and are the main reason for PLQY loss in perovskite NCs is only partially true: if one takes charge balance into account, a halide (charged) deficiency is compensated by a cation deficiency, which can emerge either from $\mathrm{Cs}$ or $\mathrm{Pb}$. This means that the depletion of halide happens in the form of $\mathrm{CsBr}$ and $\mathrm{PbBr}_{2}$, respectively. It is also important to always specify the charge of the studied defects because the energetics can be very different if they are in either the neutral or charged form. For example, a neutral $\mathrm{Br}$ vacancy has a very high defect formation energy of $4.0 \mathrm{eV}$, whereas the corresponding charged $\mathrm{Br}$ vacancy is about half that amount. Confusing one with the other may lead to erroneous explanations of defect formations.

The data presented in this work provide a qualitative description of the type of defects that can emerge in colloidal NCs. The approach presented is still limited and can be improved in the future. For example, it is desirable to analyze the defect energy formation when the surface is passivated by ligands that are used in synthesis or after synthesis via ligand exchange, e.g., oleate, alkylammonium, but also phosphonates, sulphanates, and zwitterions. It is expected that the chemical equilibrium that describes the DFE is also affected in the presence of solvent because organic ligands can be effectively stabilized by the environment, shifting the equilibrium toward the formation of the defect, significantly lowering the DFEs. Solvent effects are purposely not included in in this work, because we wanted to focus on a simple description of the DFE. Including this next layer of detail will be the purpose of future work. Finally, we did not consider spin-orbit coupling in this work because of the size of the explicit NC clusters involved that make these calculations prohibitive. This contribution can also affect the energetics significantly.

\section{ASSOCIATED CONTENT}

\section{S Supporting Information}

The Supporting Information is available free of charge on the ACS Publications website at DOI: 10.1021/acsenergylett.9b01945.

Further computational details and illustration of relaxed structures (PDF)

\section{AUTHOR INFORMATION}

\section{Corresponding Author}

*E-mail: i.a.c.infante@vu.nl, ivan.infante@iit.it. Twitter: @ NanochemistryI1.

ORCID $\odot$

Ivan Infante: 0000-0003-3467-9376

\section{Author Contributions}

S.t.B. and F.Z. contributed equally to this work.

Notes

The authors declare no competing financial interest.

\section{ACKNOWLEDGMENTS}

I.I. acknowledges The Netherlands Organization of Scientific Research (NWO) through the Innovational Research Incentive (Vidi) Scheme (Grant No. 723.013.002). The computational work was carried out on the Dutch national e-infrastructure with the support of the SURF Cooperative.

\section{REFERENCES}

(1) Krieg, F.; Ochsenbein, S. T.; Yakunin, S.; ten Brinck, S.; Aellen, P.; Süess, A.; Clerc, B.; Guggisberg, D.; Nazarenko, O.; Shynkarenko, Y.; et al. Colloidal CsPbX $3(\mathrm{X}=\mathrm{Cl}, \mathrm{Br}, \mathrm{I})$ Nanocrystals 2.0: Zwitterionic Capping Ligands for Improved Durability and Stability. ACS Energy Lett. 2018, 3 (3), 641-646.

(2) Bodnarchuk, M. I.; Boehme, S. C.; ten Brinck, S.; Bernasconi, C.; Shynkarenko, Y.; Krieg, F.; Widmer, R.; Aeschlimann, B.; Günther, D.; Kovalenko, M. V.; et al. Rationalizing and Controlling the Surface Structure and Electronic Passivation of Cesium Lead Halide Nanocrystals. ACS Energy Lett. 2019, 4, 63-74.

(3) Correa-Baena, J.-P.; Saliba, M.; Buonassisi, T.; Grätzel, M.; Abate, A.; Tress, W.; Hagfeldt, A. Promises and Challenges of Perovskite Solar Cells in Portable Applications. Science (Washington, DC, U. S.) 2017, 358 (6364), 739-744.

(4) ten Brinck, S.; Infante, I. Surface Termination, Morphology, and Bright Photoluminescence of Cesium Lead Halide Perovskite Nanocrystals. ACS Energy Lett. 2016, 1 (6), 1266-1272.

(5) Su, Y.; Chen, X.; Ji, W.; Zeng, Q.; Ren, Z.; Su, Z.; Liu, L. Highly Controllable and Efficient Synthesis of Mixed-Halide CsPbX3 (X = $\mathrm{Cl}, \mathrm{Br}, \mathrm{I})$ Perovskite QDs toward the Tunability of Entire Visible Light. ACS Appl. Mater. Interfaces 2017, 9 (38), 33020-33028.

(6) Protesescu, L.; Yakunin, S.; Bodnarchuk, M. I.; Krieg, F.; Caputo, R.; Hendon, C. H.; Yang, R. X.; Walsh, A.; Kovalenko, M. V. Nanocrystals of Cesium Lead Halide Perovskites (CsPbX 3, X $=\mathrm{Cl}$, $\mathrm{Br}$, and I): Novel Optoelectronic Materials Showing Bright Emission with Wide Color Gamut. Nano Lett. 2015, 15 (6), 3692-3696.

(7) Lao, X.; Li, X.; Ågren, H.; Chen, G. Highly Controllable Synthesis and DFT Calculations of Double/Triple-Halide CsPbX3 (X $=\mathrm{Cl}, \mathrm{Br}, \mathrm{I}$ ) Perovskite Quantum Dots: Application to Light-Emitting Diodes. Nanomaterials 2019, 9 (2), 172.

(8) Protesescu, L.; Yakunin, S.; Bodnarchuk, M. I.; Bertolotti, F.; Masciocchi, N.; Guagliardi, A.; Kovalenko, M. V. Monodisperse Formamidinium Lead Bromide Nanocrystals with Bright and Stable Green Photoluminescence. J. Am. Chem. Soc. 2016, 138 (43), 1420214205.

(9) Pan, J.; Sarmah, S. P.; Murali, B.; Dursun, I.; Peng, W.; Parida, M. R.; Liu, J.; Sinatra, L.; Alyami, N.; Zhao, C.; et al. Air-Stable Surface-Passivated Perovskite Quantum Dots for Ultra-Robust, Single- and Two-Photon-Induced Amplified Spontaneous Emission. J. Phys. Chem. Lett. 2015, 6 (24), 5027-5033.

(10) Yakunin, S.; Protesescu, L.; Krieg, F.; Bodnarchuk, M. I.; Nedelcu, G.; Humer, M.; De Luca, G.; Fiebig, M.; Heiss, W.; Kovalenko, M. V. Low-Threshold Amplified Spontaneous Emission and Lasing from Colloidal Nanocrystals of Caesium Lead Halide Perovskites. Nat. Commun. 2015, 6, 1-8.

(11) Meyns, M.; Perálvarez, M.; Heuer-Jungemann, A.; Hertog, W.; Ibáñez, M.; Nafria, R.; Genç, A.; Arbiol, J.; Kovalenko, M. V.; 
Carreras, J.; et al. Polymer-Enhanced Stability of Inorganic Perovskite Nanocrystals and Their Application in Color Conversion LEDs. ACS Appl. Mater. Interfaces 2016, 8 (30), 19579-19586.

(12) Pan, J.; Quan, L. N.; Zhao, Y.; Peng, W.; Murali, B.; Sarmah, S. P.; Yuan, M.; Sinatra, L.; Alyami, N. M.; Liu, J.; et al. Highly Efficient Perovskite-Quantum-Dot Light-Emitting Diodes by Surface Engineering. Adv. Mater. 2016, 28 (39), 8718-8725.

(13) Zhang, X.; Sun, C.; Zhang, Y.; Wu, H.; Ji, C.; Chuai, Y.; Wang, P.; Wen, S.; Zhang, C.; Yu, W. W. Bright Perovskite Nanocrystal Films for Efficient Light-Emitting Devices. J. Phys. Chem. Lett. 2016, 7 (22), 4602-4610.

(14) Chiba, T.; Hoshi, K.; Pu, Y. J.; Takeda, Y.; Hayashi, Y.; Ohisa, S.; Kawata, S.; Kido, J. High-Efficiency Perovskite Quantum-Dot Light-Emitting Devices by Effective Washing Process and Interfacial Energy Level Alignment. ACS Appl. Mater. Interfaces 2017, 9 (21), 18054-18060.

(15) Deng, W.; Xu, X.; Zhang, X.; Zhang, Y.; Jin, X.; Wang, L.; Lee, S. T.; Jie, J. Organometal Halide Perovskite Quantum Dot LightEmitting Diodes. Adv. Funct. Mater. 2016, 26 (26), 4797-4802.

(16) Li, G.; Rivarola, F. W. R.; Davis, N. J. L. K.; Bai, S.; Jellicoe, T. C.; De La Peña, F.; Hou, S.; Ducati, C.; Gao, F.; Friend, R. H.; et al. Highly Efficient Perovskite Nanocrystal Light-Emitting Diodes Enabled by a Universal Crosslinking Method. Adv. Mater. 2016, 28 (18), 3528-3534.

(17) Swarnkar, A.; Marshall, A. R.; Sanehira, E. M.; Chernomordik, B. D.; Moore, D. T.; Christians, J. A.; Chakrabarti, T.; Luther, J. M. Quantum Dot-Induced Phase Stabilization of a-CsPbI3 Perovskite for High-Efficiency Photovoltaics. Science (Washington, DC, U. S.) 2016, 354 (6308), 92-95.

(18) Wheeler, L. M.; Sanehira, E. M.; Marshall, A. R.; Schulz, P.; Suri, M.; Anderson, N. C.; Christians, J. A.; Nordlund, D.; Sokaras, D.; Kroll, T.; et al. Targeted Ligand-Exchange Chemistry on Cesium Lead Halide Perovskite Quantum Dots for High-Efficiency Photovoltaics. J. Am. Chem. Soc. 2018, 140 (33), 10504-10513.

(19) Zou, Y.; Wang, H. Y.; Qin, Y.; Mu, C.; Li, Q.; Xu, D.; Zhang, J. P. Reduced Defects of MAPbI 3 Thin Films Treated by FAI for HighPerformance Planar Perovskite Solar Cells. Adv. Funct. Mater. 2019, 29 (7), 1805810.

(20) Meggiolaro, D.; Mosconi, E.; De Angelis, F. Modeling the Interaction of Molecular Iodine with MAPbI3: A Probe of LeadHalide Perovskites Defect Chemistry. ACS Energy Lett. 2018, 3 (2), 447-451.

(21) Kang, J.; Wang, L.-W. High Defect Tolerance in Lead Halide Perovskite CsPbBr3. J. Phys. Chem. Lett. 2017, 8 (2), 489-493.

(22) Leijtens, T.; Barker, A. J.; Grancini, G.; Ball, J. M.; Kandada, A. R. S.; Petrozza, A.; Eperon, G. E.; Zhang, W.; Snaith, H. J. Carrier Trapping and Recombination: The Role of Defect Physics in Enhancing the Open Circuit Voltage of Metal Halide Perovskite Solar Cells. Energy Environ. Sci. 2016, 9 (11), 3472-3481.

(23) Adinolfi, V.; Yuan, M.; Comin, R.; Thibau, E. S.; Shi, D.; Saidaminov, M. I.; Kanjanaboos, P.; Kopilovic, D.; Hoogland, S.; Lu, Z. H.; et al. The In-Gap Electronic State Spectrum of Methylammonium Lead Iodide Single-Crystal Perovskites. Adv. Mater. 2016, 28 (17), 3406-3410.

(24) Pan, A.; He, B.; Fan, X.; Liu, Z.; Urban, J. J.; Alivisatos, A. P.; He, L.; Liu, Y. Insight into the Ligand-Mediated Synthesis of Colloidal CsPbBr 3 Perovskite Nanocrystals: The Role of Organic Acid, Base, and Cesium Precursors. ACS Nano 2016, 10 (8), 79437954.

(25) Kim, J.; Ho-Baillie, A.; Huang, S. Review of Novel Passivation Techniques for Efficient and Stable Perovskite Solar Cells. Sol. RRL 2019, 3 (4), 1800302.

(26) Connelly, N. G.; Hartshorn, R. M.; Damhus, T.; Hutton, A. T. Nomenclature of Inorganic Chemistry IUPAC Recommendations 2005; 2005; Vol. 128.

(27) Zhang, S. B.; Northrup, J. E. Chemical Potential Dependence of Defect Formation Energies in GaAs: Application to Ga Self-Diffusion. Phys. Rev. Lett. 1991, 67 (17), 2339-2342.
(28) Zhang, S. B.; Wei, S. H.; Zunger, A. Intrinsic N-Type versus pType Doping Asymmetry and the Defect Physics of ZnO. Phys. Rev. B: Condens. Matter Mater. Phys. 2001, 63 (7), 1-7.

(29) Wei, S.; Yang, Y.; Kang, X.; Wang, L.; Huang, L.; Pan, D. Room-Temperature and Gram-Scale Synthesis of $\mathrm{CsPbX} 3(\mathrm{X}=\mathrm{Cl}$, Br, I) Perovskite Nanocrystals with 50-85\% Photoluminescence Quantum Yields. Chem. Commun. 2016, 52 (45), 7265-7268.

(30) Zhang, F.; Zhong, H.; Chen, C.; Wu, X.; Hu, X.; Huang, H.; Han, J.; Zou, B.; Dong, Y. Brightly Luminescent and Color-Tunable Colloidal CH 3 NH 3 PbX 3 (X = Br, I, Cl) Quantum Dots: Potential Alternatives for Display Technology. ACS Nano 2015, 9 (4), 45334542.

(31) Hutter, J.; Iannuzzi, M.; Schiffmann, F.; Vandevondele, J. Cp2k: Atomistic Simulations of Condensed Matter Systems. Wiley Interdiscip. Rev. Comput. Mol. Sci. 2014, 4 (1), 15-25.

(32) Perdew, J. P.; Burke, K.; Ernzerhof, M. Generalized Gradient Approximation Made Simple. Phys. Rev. Lett. 1996, 77 (18), 38653868.

(33) Adamo, C.; Barone, V. Toward Reliable Density Functional Methods without Adjustable Parameters: The PBE0Model. J. Chem. Phys. 1999, 110 (13), 6158-6170.

(34) De Roo, J.; Ibáñez, M.; Geiregat, P.; Nedelcu, G.; Walravens, W.; Maes, J.; Martins, J. C.; Van Driessche, I.; Kovalenko, M. V.; Hens, Z. Highly Dynamic Ligand Binding and Light Absorption Coefficient of Cesium Lead Bromide Perovskite Nanocrystals. ACS Nano 2016, 10 (2), 2071-2081.

(35) Di Stasio, F.; Christodoulou, S.; Huo, N.; Konstantatos, G. Near-Unity Photoluminescence Quantum Yield in CsPbBr3 Nanocrystal Solid-State Films via Postsynthesis Treatment with Lead Bromide. Chem. Mater. 2017, 29 (18), 7663-7667.

(36) Imran, M.; Ijaz, P.; Goldoni, L.; Maggioni, D.; Petralanda, U.; Prato, M.; Almeida, G.; Infante, I.; Manna, L. Simultaneous Cationic and Anionic Ligand Exchange For Colloidally Stable CsPbBr 3 Nanocrystals. ACS Energy Lett. 2019, 4 (4), 819-824.

(37) Jing, Q.; Xu, Y.; Su, Y.; Xing, X.; Lu, Z. A Systematic Study of the Synthesis of Cesium Lead Halide Nanocrystals: Does Cs4PbBr6 or CsPbBr3 Form? Nanoscale 2019, 11 (4), 1784-1789.

(38) Liu, Z.; Bekenstein, Y.; Ye, X.; Nguyen, S. C.; Swabeck, J.; Zhang, D.; Lee, S.-T.; Yang, P.; Ma, W.; Alivisatos, A. P. Ligand Mediated Transformation of Cesium Lead Bromide Perovskite Nanocrystals to Lead Depleted Cs $4 \mathrm{PbBr} 6$ Nanocrystals. J. Am. Chem. Soc. 2017, 139 (15), 5309-5312.

(39) Akkerman, Q. A.; Park, S.; Radicchi, E.; Nunzi, F.; Mosconi, E.; De Angelis, F.; Brescia, R.; Rastogi, P.; Prato, M.; Manna, L. Nearly Monodisperse Insulator Cs4PbX6 $(\mathrm{X}=\mathrm{Cl}, \mathrm{Br}, \mathrm{I})$ Nanocrystals, Their Mixed Halide Compositions, and Their Transformation into CsPbX3 Nanocrystals. Nano Lett. 2017, 17 (3), 1924-1930.

(40) Udayabhaskararao, T.; Houben, L.; Cohen, H.; Menahem, M.; Pinkas, I.; Avram, L.; Wolf, T.; Teitelboim, A.; Leskes, M.; Yaffe, O.; et al. A Mechanistic Study of Phase Transformation in Perovskite Nanocrystals Driven by Ligand Passivation. Chem. Mater. 2018, 30 (1), 84-93. 